\title{
Zur Geschichte der MGM/MGZ
}

Klaus A. Maier

Persönliche Erinnerungen an eine 20-jährige Redaktionstätigkeit für die "Militärgeschichtlichen Mitteilungen«

https://doi.org/10.1515/mgzs-2017-0153

Im Frühjahr 1973 wurde ich von den Redakteuren Wilhelm Deist (Dokumentationen) und Horst Zoske (Anzeigen/Rezensionen) als Nachfolger für Johannes Fischer (Aufsätze) in das dreiköpfige Redaktionskollegium der »Militärgeschichtlichen Mitteilungen« (MGM) kooptiert und war ununterbrochen bis Ende 1993 für die Auswahl und redaktionelle Betreuung der Aufsätze verantwortlich. Diese lange Stehzeit der MGM-Redakteure für die Großbeiträge war üblich, lediglich im arbeitsreichsten Anzeigen- und Rezensionsgeschäft fand öfter ein Wechsel statt. Das Interesse der Kollegen des Militärgeschichtlichen Forschungsamtes (MGFA) an der zeitaufwändigen Nebentätigkeit für die MGM war nicht besonders ausgeprägt, sodass die Suche nach einem Nachfolger nicht immer einfach war.

Bei der Auswahl der Aufsätze konnte ich all die Jahre hindurch auf ein Überangebot an guten Aufsätzen zurückgreifen, von denen allerdings nur sechs bis maximal acht pro Jahrgang veröffentlicht werden konnten. Die sachlich zu begründende Ablehnung eines Angebots erforderte bisweilen mehr zeitlichen und argumentativen Aufwand als die Bearbeitung angenommener Beiträge. Da die Mehrzahl der angebotenen Aufsätze zeitgeschichtliche Themen behandelte, war das Vorhaben der Redaktion, in den MGM alle Epochen der Militärgeschichte abzudecken, nur eingeschränkt umsetzbar.

Die für die Publikation ausgewählten Aufsätze, Dokumentationen, Literaturberichte und Anzeigen/Rezensionen wurden von allen Redakteuren gelesen, schriftlich kommentiert und nach einstimmiger Befürwortung als Programm für ein bestimmtes Heft den Herausgebern, dem Amtschef und dem Leitenden Historiker, zum Imprimatur vorgelegt.

Für die Redaktion war Militärgeschichte eine Spezialdisziplin der Geschichtswissenschaft. Dieser methodische Anspruch bildete das Hauptkriterium für die

Kontakt: Klaus A. Maier, Schliengen, E-Mail: klaus.autbert.maier@t-online.de 
Auswahl der Beiträge. Die MGM sollten gemäß der Hausverfügung Nr. 133/66 vom 3. Oktober 1966 als Kommunikationsplattform zwischen dem MGFA und der historischen Fachwelt dienen. Gleichzeitig leisteten sie einen Beitrag zur historischen Bildung in der Bundeswehr. Dazu wurden die MGM bis auf die Bataillonsebene hinab verteilt. Gelegentliche Nachforschungen ergaben, dass die MGM dort nicht selten in den Vorschriftenstellen ungelesen ihr Grab fanden.

Mit ihrem Wissenschaftsverständnis geriet die Redaktion in Konflikt mit Oberst i.G. Othmar Hackl, Amtschef vom 1. Oktober 1976 bis 31. März 1985. Hackl wollte die »Hauptkommunikationsrichtung « des MGFA vermehrt auf die Belange der Bundeswehr umlenken und ließ »kriegsgeschichtliche Beispiele aus dem Zweiten Weltkrieg für die taktische Führerausbildung und -weiterbildung im Heer « bearbeiten. ${ }^{1}$ Dieses applikatorische Erkenntnisinteresse hatte der erste Amtschef des MGFA, Oberst i.G. Hans Meier-Welcker, wegweisend für das MGFA stets als unhistorisch abgelehnt. ${ }^{2}$ In diesen Studien wurde die Wehrmacht, die Hackl für die Tradition der Bundeswehr retten wollte, weniger kritisch dargestellt, als es die Forschungsergebnisse des MGFA lange vor den Wanderausstellungen des Hamburger Instituts für Sozialforschung über die Verbrechen der Wehrmacht (1995 bis 1999 und 2001 bis 2004) nahelegten. ${ }^{3}$ In der Folge hatte die MGM-Redaktion immer dann große Schwierigkeiten, vom Amtschef das Imprimatur für Beiträge zu erhalten, wenn in ihnen die Wehrmacht oder einzelne ihrer Soldaten kritisch behandelt wurden. Ich erinnere mich vor allem an heftige Diskussionen, die dem Imprimatur für die Aufsätze von Gerhard L. Weinberg »Zur Dotation Hitlers an Generalfeldmarschall Ritter von Leeb« (MGM, 26 [1979], 2) und Christopher R. Browning »Wehrmacht Reprisal Policy and the Mass Murder of Jews in Serbia« (MGM, 33 [1983], 1) vorausgingen.

Weinberg widerlegt die in einer MGFA-Publikation aufgestellte Behauptung, wonach die Dotation Hitlers für Leeb vom September 1941 in Höhe von

1 Zit. nach: Vorwort des Amtschefs. In: Militärgeschichte. Probleme - Thesen - Wege. Im Auftrag des Militärgeschichtlichen Forschungsamtes aus Anlaß seines 25jährigen Bestehens ausgewählt und zusammengestellt von Manfred Messerschmidt, Klaus A. Maier, Werner Rahn und Bruno Thoß, Stuttgart 1982 (= Beiträge zur Militär- und Kriegsgeschichte, 25), S. 10.

2 Hans Meier-Welcker, Unterricht und Studium in der Kriegsgeschichte angesichts der radikalen Wandlung im Kriegswesen. In: Wehrkunde, 9 (1960), S. 680-612, wieder abgedruckt in Militärgeschichte (wie Anm. 1), S. 18-21. Siehe dazu Klaus A. Maier, Von der Generalstabswissenschaft zur modernen Militärgeschichte. Hans Meier-Welcker und die Anfänge der militärgeschichtlichen Bildung in der Bundeswehr (in Vorb.).

3 Siehe dazu Klaus A. Maier, Überlegungen zur Zielsetzung und Methode der Militärgeschichtsschreibung im Militärgeschichtlichen Forschungsamt und die Forderung nach deren Nutzen für die Bundeswehr seit der Mitte der 70er Jahre. In: MGM, 52 (1993), 2, S. 360-370, hier S. 365f. 
250000 Reichsmark diesen »verwirrt« und »bestürzt« habe. ${ }^{4}$ Er beschreibt, wie Leeb in seinem Bemühen um den Erwerb eines Waldgrundstückes bei Passau schließlich eine Dotation im Wert von insgesamt 880000 Reichsmark erhielt. Der Generalfeldmarschall bedankte sich dafür am 26. Juli 1944 bei Hitler und nutzte diese Gelegenheit, ihm »für die wunderbare Errettung vor dem ruchlosen Anschlage« seine »tiefgefühlten Glückwünsche auszusprechen «. ${ }^{5}$

Nur mühsam konnte dem Amtschef sein Vorhaben ausgeredet werden, künftig keine Amtspublikationen mehr in den MGM rezensieren oder gar kritisieren zu lassen. Dies hätte zu der unfairen Situation geführt, in der MGFA-Mitarbeiter zwar weiterhin auswärtige Historiker hätten kritisieren dürfen, selbst aber von Kritik in ihrer Hauszeitschrift verschont geblieben wären. Eine solche Regelung hätte darüber hinaus bedeutet, dass MGFA-Publikationen, also ein wichtiges Segment der deutschen militärhistorischen Forschung, der MGM-Leserschaft nicht mehr vorgestellt worden wären. Für die MGM-Redaktion hatten das historische Erkenntnisinteresse und der offene wissenschaftliche Diskurs Vorrang vor der Rücksichtnahme auf den Betriebsfrieden im MGFA.

Browning untersucht die Beteiligung der Wehrmacht an der Ermordung der männlichen Juden in Serbien im Herbst 1941, also noch bevor der planmäßige Genozid an den europäischen Juden im Gange war. ${ }^{6}$ Von besonderer Brisanz war, dass der damalige Chef des Stabes des XVIII. Gebirgskorps, Max-Josef Pemsel, in dieses Verbrechen involviert war. Pemsel wurde 1956 als Generalmajor in die Bundeswehr übernommen, war Befehlshaber im Wehrbereich V (München), ab April 1957 Kommandierender General des II. Korps in Ulm und wurde im September 1961 als Generalleutnant in den Ruhestand versetzt.

Am 29. Juli 1983 »meldete« Hackl seinem Dienstvorgesetzten, dem Stellvertreter des Generalinspekteurs der Bundeswehr, dass der Leitende Historiker, Manfred Messerschmidt, der der Veröffentlichung des Aufsatzes zugestimmt hatte,

\footnotetext{
4 Zit. nach: Generalfeldmarschall Ritter von Leeb. Tagebuchaufzeichnungen und Lagebeurteilungen aus zwei Weltkriegen. Aus dem Nachlaß hrsg. und mit einem Lebensabriß versehen von Georg Meyer, Stuttgart 1976 (= Beiträge zur Militär- und Kriegsgeschichte, 16), S. 65 f.

5 Zit. nach: Gerhard L. Weinberg, Zur Dotation Hitlers an Generalfeldmarschall Ritter von Leeb. In: MGM, 26 (1979), 2, S. 98.

6 Am 2.4.1941 ergänzten der Chef des Generalstabes des Heeres, Franz Halder, und sein Generalquartiermeister, Eduard Wagner, den Befehlsentwurf vom 26.3.1941 über die Rolle der »Sonderkommandos der Sicherheitspolizei (SD)« im rückwärtigen Heeresgebiet gegen »führende Emigranten, Saboteure, Terroristen usw.« für den Balkan um zwei weitere Gegner, nämlich »Kommunisten, Juden«. Zit. nach: Jürgen Förster, Das Unternehmen »Barbarossa» als Eroberungs- und Vernichtungskrieg. In: Der Angriff auf die Sowjetunion. Hrsg. vom Militärgeschichtlichen Forschungsamt, Stuttgart 1983 (= Das Deutsche Reich und der Zweite Weltkrieg, 4), S. 423.
} 
»Reibungen im Zusammenhang mit dem Beitrag von Ch. Browning für die MGM [...] verursacht hat «. ${ }^{7}$

Dass der Amtschef am Ende doch noch sein Imprimatur für die Aufsätze von Weinberg und Browning erteilte, ist letztlich darauf zurückzuführen, dass ihm die Redaktion für den Fall einer Ablehnung dieser Beiträge nachdrücklich die drohende Beschädigung der hohen internationalen Reputation der MGM als wissenschaftliches »Aushängeschild« des MGFA vor Augen führte.

Auch in die Organisation der MGM-Redaktion griff Hackl ein. Seiner Vorstellung von der unteilbaren militärischen Führungsverantwortung waren Verhandlungen mit einem Kollegium fremd. Er setzte deshalb den Leiter der Schriftleitung als vierten Redakteur mit alleinigem Vortragsrecht beim Amtschef ein. Meiner Erinnerung nach hat der damalige Schriftleiter, Friedrich Freiherr Hiller von Gaertringen, das von der Redaktion beschlossene Veröffentlichungsprogramm beim Amtschef stets loyal vertreten. Diese Erweiterung der Redaktion um den Schriftleiter wurde von den nachfolgenden Amtschefs wieder aufgehoben.

Die beschriebenen Schwierigkeiten waren zeitlich begrenzt und konnten mit der uneingeschränkten Solidarität meiner Redaktionskollegen gemeistert werden. Sie beeinträchtigen nicht meine insgesamt sehr positiven Erinnerungen an meine Zeit in der MGM-Redaktion. Die neben meiner Mitarbeit an verschiedenen Forschungsprojekten des MGFA zu leistende Redaktionstätigkeit bescherte mir zahlreiche fruchtbare Kontakte $\mathrm{zu}$ in- und ausländischen Kollegen und erweiterte meinen historischen und methodischen Wissenshorizont.

7 MGFA, Amtschef an Stellvertreter des Generalinspekteurs der Bundeswehr, 29.7.1983, BArch, BW/2, Bd 18467. 